\title{
Synthesis and Cytotoxicity of Novel Pyrazole Derivatives Derived from 3-Methyl-1-phenyl-1H- pyrazol-5(4H)-one
}

\section{Elham Ezz El-Arab}

National Organization for Drug Control \& Research-P.O. Box29, Cairo, Egypt.

\begin{abstract}
T HE 3-Methyl-1-phenyl-1H-pyrazol-5(4H)-one (1) was used to synthesis pyran,and pyridine derivatives through its multicomponent reactions with aromatic aldehydes and cyanomethylene reagents. The synthesized products were evaluated for their cytotoxicity against cancer and normal cell lines. 4a, 4c, 6c, 7c, 11b, $11 \mathrm{c}$ and 13 showed optimal cytotoxicity among the tested compounds. The toxicity of the most potent cytotoxic compounds was measured using Brine-Shrimp Lethality Assay.
\end{abstract}

Keywords: Pyrazole, Pyran, Pyridine, Cytotoxicity and Toxicity.

Multicomponent reactions (MCRs) have emerged as a valuable tool in the preparation of structurally diverse chemical libraries of heterocyclic compounds $^{(1)}$. They are inherently atom economical processes in which relatively complex products can be obtained in a one-pot reaction from simple starting materials, and thus they exemplify many of the desired features of an ideal synthesis. MCRs are generally much more environmentally friendly and offer access to large compound libraries with diverse functionalities with the avoidance of protection and deprotection steps for possible combinatorial surveying of structural variations. In view of the increasing interest in the preparation of a large variety of heterocyclic compound libraries, the development of new synthetically valuable MCRs with several diversity points remains a challenge for both academic and industrial institutions ${ }^{(2)}$. Thiophene and its derivatives are an important class of heterocyclic compounds possessing broad biological activities, such as anti-inflammatory ${ }^{(3)}$, analgesic ${ }^{(3)}$, antioxidant ${ }^{(4)}$, antitubercular ${ }^{(5)}$, antidepressant ${ }^{(6)}$, sedative ${ }^{(6)}$, antiamoebic ${ }^{(7)}$, oral analgesic ${ }^{(8)}$, anti-metabolite ${ }^{(9)}$ and antineoplastic properties ${ }^{(10)}$. Many pyrazole derivatives have attracted considerable attention in the recent years for their diverse biological activities ${ }^{(11-16)}$. They are also acknowledged for their anticancer activities (17-19). Celecoxib (1), Sulfaphenazole (2), CDPPB (3), Linazolac (4), Mepiprazole (5), and Rimonabant (6) are some of the pyrazole based drugs available today in the market (Fig. 1) ${ }^{(20)}$.

From the aforementioned reports, it seemed that the development of an efficient, rapid and clean synthetic route towards focused libraries of such 
compounds is of great importance to both medicinal and synthetic chemists. Hence in this work, we report a one-pot, three-component reaction for the synthesis of pyran derivatives through the reaction of 3-methyl-phenyl-pyrazol$5(4 \mathrm{H})$-one (1) with different aromatic aldehydes and cyanomethylene reagents . All the synthesized compounds were characterized using FT-IR, ${ }^{1} \mathrm{H}$ NMR and mass spectrometry and were subjected to screening towards cancer cell lines.

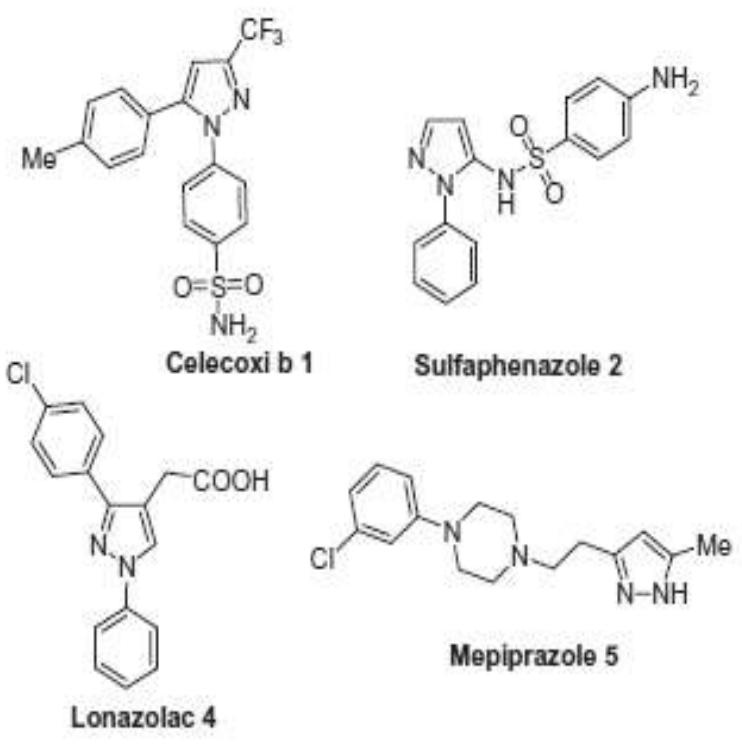

Fig.1. Biologically active pyrazole derivatives.

\section{Chemistry}

\section{Results and Discussion}

The present investigation emphasized mainly on the synthesis of molecules derived from of 3-methyl-1-phenyl-1H-pyrazol-5(4H)-one (1) and evaluation of their cytotoxicity against cancer and normal cell lines. The synthetic strategies adopted for the synthesis of the intermediate and target compounds are depicted in Schemes $1 \& 2$. One pot multi-component reactions (MCR) were utilized to prepare the target compounds. The reaction of the 3-methyl-1-phenyl-1Hpyrazol-5(4H)-one (1) with each of benzaldehyde (2a), 4-methoxybenzaldehyde (2b) or 4-chlorobenzaldehyde (2c) and ethyl cyanoacetate (3) afforded the 6oxopyranopyrazole derivatives $4 \mathrm{a}-\mathrm{c}$. Structures of the latter products were confirmed on the basis of their respective analytical and spectral data. Thus, ${ }^{1} \mathrm{H}$ NMR spectrum of compound $4 \mathrm{a}$ revealed the presence of a singlet $\delta$ at $2.88 \mathrm{ppm}$ for $\mathrm{CH}_{3}$ and a multiplet $\delta 7.48-8.09$ ppm corresponding to two phenyl protons. Meanwhile, the reaction of compound 1 with either of $2 a, 2 b$ or $2 c$ and malononitrile (5) in ethanol containing triethylamine gave the 6-Amino-3-

Egypt. J. Chem. 58, No. 6 (2015) 
methyl-4-aryl-1,4-dihydropyrano [2,3-c] pyrazole-5- carbonitrile derivatives 6a$c$, respectively. The analytical and spectral data of $6 \mathrm{a}-\mathrm{c}$ were in consistence with their respective structures. Compounds 6a-c reacted with hydrazine hydrate in 1,4-dioxane solution containing sodium acetate to give the 5-amino-3-methyl-4aryl-4,7-dihydro-1H-pyrano[2,3-c:6,5-c'] dipyrazol--3-amine derivatives 7a-c. The structures of the latter products were based on their respective analytical and spectral data. On the other hand, the reaction of compound 1 with pyridine-3aldehyde (8) and malononitrile afforded the 6-amino-3-methyl-4-(pyridin-3-yl)1,4-dihydropyrano [2,3-c]pyrazole-5-carbonitrile (9). The structure of the latter product was based on its respective analytical and spectral data. Thus, the ${ }^{1} \mathrm{H}$ NMR spectrum showed the presence of singlet at $\delta 1.83 \mathrm{ppm}$ corresponding to $\mathrm{CH}_{3}$ group, a singlet at $4.63 \mathrm{ppm}$ corresponding for pyrane proton and a singlet $\delta 6.82 \mathrm{ppm}$ for $\mathrm{NH}_{2}$ - and a multiplet $\delta 7.30-8.39 \mathrm{ppm}$ for phenyl and pyridine protons.

Moreover, the reaction of 1 with the aromatic aldehydes 2a-c and 2aminoprop-1-ene-1,1,3-tricarbonitrile (10) in ethanol containing a catalytic amount of triethylamine afforded the pyrazolopyrano[2,3-b]pyridine-6carbonitrile derivatives 11a-c. ${ }^{1} \mathrm{H}$ NMR of compound 11a (as an example) showed the presence of a singlet $\delta 2.78 \mathrm{ppm}$ for $\mathrm{CH}_{3}$ group, a singlet $\delta 4.58$ ppm for pyrane proton, two singlet $\delta 4.16-4.88 \mathrm{ppm}$ corresponding to two $\mathrm{NH}_{2}$ protons and a multiplet $\delta 7.28-7.73$ ppm for phenyl protons .

Moreover, On the other hand, the reaction of compound $6 \mathrm{~b}$ with phenylisothiocyanate (12) in ethanol afforded the corresponding thiourea derivative 13 , the structure of which was based on analytical and spectral data.

The one-pot reaction of compound 1 with salicylaldehyde and malononitrile gave the annulated 5-Amino-1-methyl-3H-chromeno[4',3':4,5]-pyrano[2,3c]pyrazol-6(11bH)-one (14). The analytical and spectral data of the latter product was the basis of their structural elucidation. Thus, the ${ }^{1} \mathrm{H}$ NMR spectrum of 14 showed, beside the expected signals, the presence of a singlet at $\delta 2.76$ ppm for $\mathrm{CH}_{3}$, singlet at $\delta 4.48$ pyran $\mathrm{H}-4$, a singlet at $\delta 4.26$ corresponding to $\mathrm{NH}_{2}$ group and a multiplet at $\delta$ 7.27-7.42 for two phenyl protons.

\section{In vitro cytotoxicity}

Effect on the growth of human cancer cell lines

The heterocyclic compounds, prepared in this study, were evaluated according to standard protocols for their in vitro cytotoxicity against six human cancer cell lines including cells derived from human gastric cancer (NUGC), human colon cancer (DLD1), human liver cancer (HA22T and HEPG2), nasopharyngeal carcinoma (HONE1), human breast cancer (MCF) and normal fibroblast cells (WI38). (For comparison reasons, CHS 828 was used as standard anticancer drug. All of $\mathrm{IC}_{50}$ values in $(\mathrm{nM})$ are listed in Table 1 . Many of the synthesized heterocyclic compounds were observed with significant cytotoxicity 
against most of the cancer cell lines tested $\left(\mathrm{IC}_{50}<1000 \mathrm{nM}\right)$. Normal fibroblasts cells (WI38) were affected to a much lesser extent $\left(\mathrm{IC}_{50}>10,000 \mathrm{nM}\right)$. Among the tested compounds the 4-(4-Chlorophenyl)-5-methyl -7- phenyl- 4, 7- dihydro1H-pyrano [2,3-c:6,5-c'] dipyrazol-3-amine (7c) was found to show the highest cytotoxic effect against the six cancer cell lines in the range of $\mathrm{IC}_{50} 63-1088 \mathrm{nM}$. Broad spectrum antitumor activity was exhibited by compounds $4 a, 4 c, 6 c, 7 c$, $11 \mathrm{~b}, 11 \mathrm{c}$ and 13 .
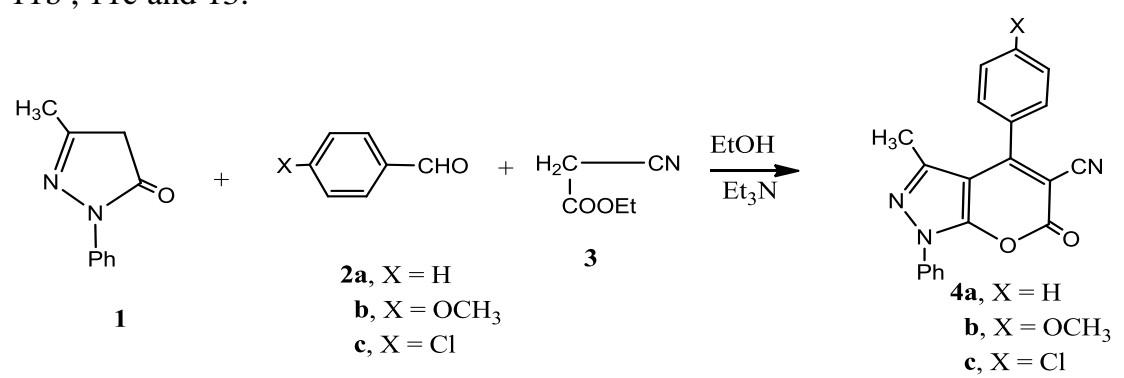

$$
\text { 6a-c }
$$

Scheme 1

Egypt. J. Chem. 58, No. 6 (2015) 


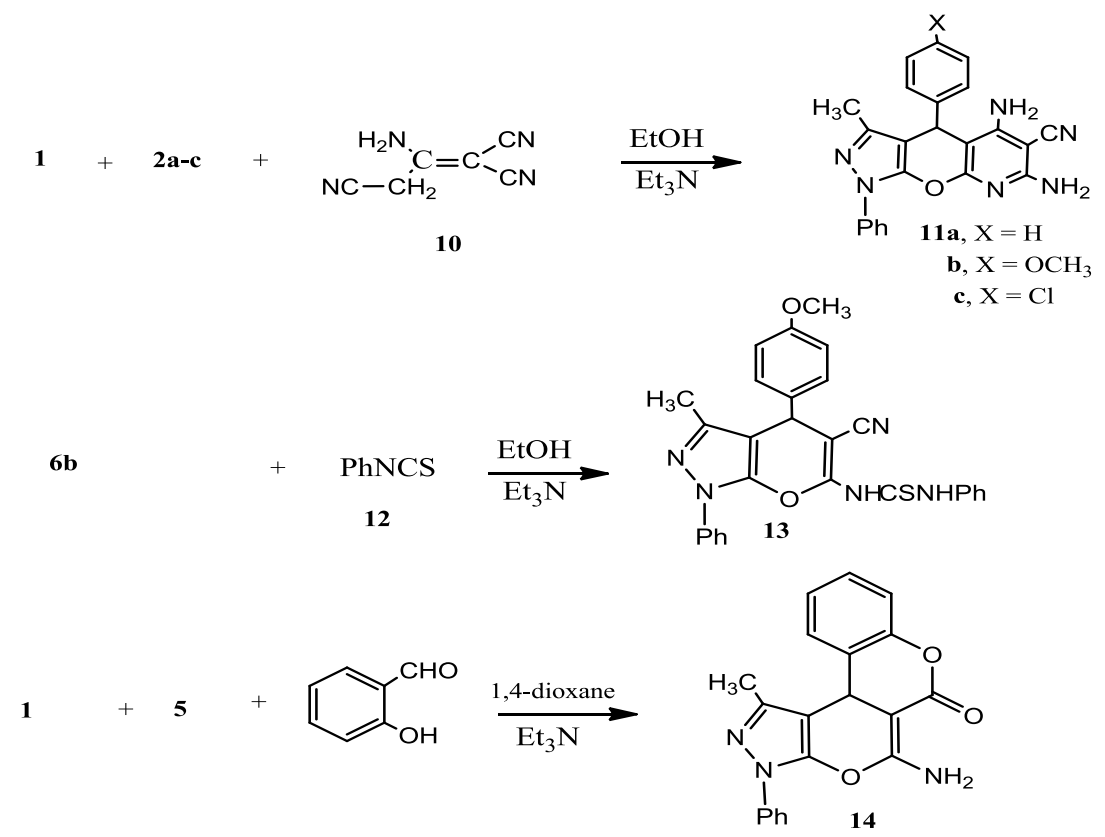

Scheme 2

TABLE 1. Cytotoxicity of the synthesized compounds against a variety of cancer cell lines ${ }^{\mathrm{a}}\left[\mathrm{IC}_{50} \mathrm{~b}(\mathrm{nM})\right]$.

\begin{tabular}{|c|c|c|c|c|c|c|c|}
\hline \multirow{2}{*}{ Compd } & \multicolumn{7}{|c|}{ Cytotoxocity $\left(\mathrm{IC}_{50}\right.$ in $\left.\mathrm{nM}\right)$} \\
\hline & NUGC & DLDI & HA22T & HEPG 2 & HONE1 & MCF & WI38 \\
\hline $4 \mathrm{a}$ & 343 & 440 & 120 & 415 & 527 & 231 & $\mathrm{Na}$ \\
\hline $4 \mathrm{~b}$ & 1280 & 2237 & 2337 & 428 & 1168 & 580 & $\mathrm{Na}$ \\
\hline $4 \mathrm{c}$ & 60 & 220 & na & 227 & 2354 & 228 & $\mathrm{Na}$ \\
\hline $6 \mathrm{a}$ & 1084 & 890 & 3068 & 399 & 2280 & 3365 & $\mathrm{Na}$ \\
\hline $6 b$ & 2420 & 2445 & 3017 & 2320 & 1820 & 3444 & $\mathrm{Na}$ \\
\hline $6 c$ & 210 & 120 & 283 & 359 & 206 & 2655 & $\mathrm{Na}$ \\
\hline $7 \mathrm{a}$ & 2219 & 2118 & 1268 & 2092 & 1255 & 1893 & 2297 \\
\hline $7 \mathrm{~b}$ & 1279 & 230 & 84 & 2489 & 2140 & 1177 & $\mathrm{Na}$ \\
\hline $7 \mathrm{c}$ & 63 & 28 & 166 & 1088 & 208 & 38 & $\mathrm{Na}$ \\
\hline 9 & 1101 & 1180 & 58 & 2766 & 180 & $\mathrm{Na}$ & $\mathrm{Na}$ \\
\hline $11 \mathrm{a}$ & 3124 & 2670 & 1165 & 4321 & 2166 & 112 & $\mathrm{Na}$ \\
\hline $11 \mathrm{~b}$ & 122 & 90 & 212 & 440 & 1877 & 436 & $\mathrm{Na}$ \\
\hline $11 \mathrm{c}$ & 40 & 60 & 152 & 320 & 2280 & 1663 & 453 \\
\hline 13 & 36 & 326 & 122 & 421 & 682 & 1293 & 1231 \\
\hline 14 & 3255 & 2674 & 1374 & 2693 & 2227 & 1438 & $\mathrm{Na}$ \\
\hline CHS 828 & 25 & 2315 & 2067 & 1245 & 15 & 18 & $\mathrm{Na}$ \\
\hline
\end{tabular}

${ }^{a}$ NUGC, gastric cancer; DLDI, colon cancer; HA22Tand HEPG2, liver cancer; HONEI, nasopharyngeal carcinoma; MCF, breast cancer; WI38, normal fibroblast cells.

${ }^{\mathrm{b}}$ The sample concentration that produces a 50\% reduction in cell growth. 
Structure activity relationship

In the present study, a series of heterocyclic derivatives incorporating a pyrazole moiety were synthesized and evaluated for their cytotoxicty aiming at investigating their SAR. Thus 6-oxopyranopyrazole derivatives 4a-c and their amino analogs 6a-c and 9 were prepared. Referring to the $\mathrm{IC}_{50}$ values listed in Table 1, compound 4a bearing a phenyl substituent exhibited significant broad spectrum cytotoxic activity in the range of $\left(\mathrm{IC}_{50} 120-527 \mathrm{nM}\right)$. Meanwhile, $4 \mathrm{~b}$ bearing a 4- $\mathrm{OCH}_{3} \mathrm{C}_{6} \mathrm{H}_{4}$ group showed selective activity against liver cancer HEPG2 $\left(\mathrm{IC}_{50} 428 \mathrm{nM}\right)$ and breast cancer MCF $\left(\mathrm{IC}_{50} 580 \mathrm{nM}\right)$. The $4-\mathrm{ClC}_{6} \mathrm{H}_{4}$ substituted derivative $4 \mathrm{c}$ demonstrated better activity compared to $4 \mathrm{a}$ and $4 \mathrm{~b}$ especially against gastric cancer NUGC $\left(\mathrm{IC}_{50} 60 \mathrm{nM}\right)$. Among the 6-amino-4substituted pyranopyrazole derivatives $6 \mathrm{a}-\mathrm{c}$ and 9 , derivative 6a carrying a 4$\mathrm{C}_{6} \mathrm{H}_{5}$ group was found to have selective activity against the human liver cancer cell line HEPG2 ( IC $\left._{50} 399 \mathrm{nM}\right)$ and colon cancer cell line DLDI $\left(\mathrm{IC}_{50} 890 \mathrm{nM}\right)$. However compound $6 \mathrm{~b}$ bearing $4-\mathrm{OCH}_{3} \mathrm{C}_{6} \mathrm{H}_{4}$ group was completely devoid of cytotoxic activity. On the other hand, compound $6 \mathrm{c}$ bearing the $4-\mathrm{ClC}_{6} \mathrm{H}_{4}$ moiety showed high activity against all cancer cell lines except breast cell line MCF in the range of $\left(\mathrm{IC}_{50} 120-359 \mathrm{nM}\right)$. The presence of pyridine ring in compound 9 is most probably responsible for its high potency against human liver cancer cell line HA22T $\left(\mathrm{IC}_{50} 58 \mathrm{nM}\right)$ and nasopharyngeal cancer cell line HONE1 $\left(\mathrm{IC}_{50} 180\right.$ $\mathrm{nM})$. The previous results suggest that the replacement of the 6-amino group in compounds 6a-c by a 6-oxo group in compounds $4 \mathrm{a}-\mathrm{c}$ in the latter pyranopyrazole derivatives led to compounds with enhanced cytotoxic effect which might be attributed the presence of the electronegative oxygen moiety. Meanwhile, replacement of the 2-amino group of compound $6 \mathrm{~b}$ by a phenylthiourea moiety afforded compound 13 which demonstrated a dramatic increase in the cytotoxic activity with the highest activity exhibited against NUGC $\left(\mathrm{IC}_{50} 36 \mathrm{nM}\right)$.

The investigation of the cytotoxicity of the pyrazolo [4',3':5,6]pyrano[2,3$b$ ]pyridine derivatives $11 \mathrm{a}-\mathrm{c}$ revealed that compound $11 \mathrm{a}$ bearing a phenyl group exhibited selective activity against $\mathrm{MCF}\left(\mathrm{IC}_{50} 112 \mathrm{nM}\right)$. On the other hand, compound $11 \mathrm{~b}$ bearing the $4-\mathrm{OCH}_{3} \mathrm{C}_{6} \mathrm{H}_{4}$ group was found to be active against most cancer cell lines with the highest activity against NUGC $\left(\mathrm{IC}_{50}\right.$ $122 \mathrm{nM}$ ) and DLDI $\left(\mathrm{IC}_{50} 90 \mathrm{nM}\right)$. The $4-\mathrm{ClC}_{6} \mathrm{H}_{4}$ substituted derivative $11 \mathrm{c}$ showed high cytotoxic activity against four cancer cell lines with potent activity against NUGC $\left(\mathrm{IC}_{50} 40 \mathrm{nM}\right)$ and DLDI $\left(\mathrm{IC}_{50} 60 \mathrm{nM}\right)$.

\section{Chemistry}

\section{Experimental}

All melting points were determined on a Stuart apparatus and the values given are uncorrected. IR spectra $\left(\mathrm{KBr}, \mathrm{cm}^{-1}\right)$ were determined on a Shimadzu IR 435 spectrophotometer (Faculty of Pharmacy, Cairo University, Egypt). ${ }^{1} \mathrm{H}$ NMR and ${ }^{13} \mathrm{C}$ NMR spectra were recorded on Varian Gemini $300 \mathrm{MHz}$ (Microanalysisl Center, Cairo University, Egypt) and Bruker Ascend $400 \mathrm{MHz}$ spectrophotometers (Microanalytical Unit, Faculty of Pharmacy, Cairo

Egypt. J. Chem. 58, No. 6 (2015) 
University, Egypt) using TMS as internal standard. Chemical shift values are recorded in ppm on $\delta$ scale. Mass spectra were recorded on a Hewlett Packard 5988 spectrometer (Microanalysis Center, Cairo University, Egypt). Elemental analyses were carried out at the Microanalysis Center, Cairo University, Egypt; found values were within $\pm 0.35 \%$ of the theoretical ones. Progress of the reactions was monitored using thin layer chromatography (TLC) sheets precoated with UV fluorescent silica gel Merck 60F 254 and were visualized using UV lamp.

General procedure for synthesis of compounds $4 a-c$ and $6 a-c$

To a solution of compound $1(1.74 \mathrm{~g}, 0.01 \mathrm{~mol})$ and the appropriate aldehyde $(0.01 \mathrm{~mol})$ in ethanol $(30 \mathrm{ml})$ containing triethylamine $(1.0 \mathrm{ml})$ either malononitrile $(0.66 \mathrm{~g}, 0.01 \mathrm{~mol})$ or ethyl cyanoacetate $(1.13 \mathrm{~g}, 0.01 \mathrm{~mol})$ was added. The reaction mixture, in each case, was heated under reflux for $1 \mathrm{hr}$, left to cool and the formed solid product, in each case, was collected by filtration and crystallized from ethanol.

4-Phenyl-3-methyl-6-oxo-1- phenyl-1,6- dihydropyrano [2,3-c]pyrazole-5carbonitrile (4a): Yield: $80 \%$; m.p.: $220-238{ }^{\circ} \mathrm{C}$; IR $\left(\mathrm{KBr}, \mathrm{cm}^{-1}\right)$ v: $3036(\mathrm{CH}$ aromatic), 2980, 2959 (CH aliphatic), $2223(\mathrm{CN}), 1702$ (C=O); ${ }^{1} \mathrm{H}$ NMR (DMSO-d $\left.d_{6}\right) \delta: 2.88\left(\mathrm{~s}, 3 \mathrm{H}, \mathrm{CH}_{3}\right), 7.48-8.09\left(\mathrm{~m}, 10 \mathrm{H}, 2 \mathrm{C}_{6} \mathrm{H}_{5}\right)$. MS $(\mathrm{m} / z, \%): 328$ $\left(\mathrm{M}^{+}, 42\right)$. Anal. calculated. for $\mathrm{C}_{20} \mathrm{H}_{13} \mathrm{~N}_{3} \mathrm{O}_{2}$ : $\mathrm{C}, 73.39 ; \mathrm{H}, 4.00 ; \mathrm{N}, 12.84$. Found: C, 73.22; H, 3.91; N, 12.66 .

4-(4-methoxyphenyl)-3- methyl-6-oxo-1- phenyl-1,6- dihydropyrano-[2,3clpyrazole-5- carbonitrile (4b): Yield: 70\%; m.p.: 191-193 ${ }^{\circ} \mathrm{C}$; IR $\left(\mathrm{KBr}, \mathrm{cm}^{-1}\right)$ v: 3053 (CH aromatic), 2956, $2935\left(\mathrm{CH}\right.$ aliphatic), $2220(\mathrm{CN}), 1720(\mathrm{C}=\mathrm{O}) ;{ }^{1} \mathrm{H}$ NMR (DMSO- $\left.d_{6}\right) \delta: 2.83\left(\mathrm{~s}, 3 \mathrm{H}, \mathrm{CH}_{3}\right), 3.89\left(\mathrm{~s}, 3 \mathrm{H}, \mathrm{OCH}_{3}\right), 6.88-8.32(\mathrm{~m}, 9 \mathrm{H}$, $\left.\mathrm{C}_{6} \mathrm{H}_{5}, \mathrm{C}_{6} \mathrm{H}_{4}\right)$; MS $(\mathrm{m} / z, \%): 358\left(\mathrm{M}^{+}, 28\right)$. Anal. calculated. for $\mathrm{C}_{21} \mathrm{H}_{15} \mathrm{~N}_{3} \mathrm{O}_{3}: \mathrm{C}$, 70.59; H, 4.20; N, 11.76. Found: C, 70.34; H, 4.28; N, 11.93.

4-(4-chlorophenyl)-3- methyl-6-oxo-1- phenyl-1,6- dihydropyrano [2,3-c] pyrazole-5- carbonitrile (4c): Yield: $83 \%$; m.p.:220-224 ${ }^{\circ} \mathrm{C} ; \quad \operatorname{IR}\left(\mathrm{KBr} \mathrm{cm}^{-1}\right) \mathrm{v}$ : 3036 (CH aromatic), 2963 (CH aliphatic), $2220(\mathrm{CN}), 1690(\mathrm{C}=\mathrm{O}) ;{ }^{1} \mathrm{H}$ NMR (DMSO- $d_{6}$ ) $\delta: 2.89\left(\mathrm{~s}, 3 \mathrm{H}, \mathrm{CH}_{3}\right), 7.25-7.48\left(\mathrm{~m}, 9 \mathrm{H}, \mathrm{C}_{6} \mathrm{H}_{5}, \mathrm{C}_{6} \mathrm{H}_{4}\right)$; Anal. calculated. for $\mathrm{C}_{20} \mathrm{H}_{12} \mathrm{ClN}_{3} \mathrm{O}_{2}$ : C, 66.39; H, 3.32; N, 11.62. Found: C, 66.38; H, $3.49 ; \mathrm{N}, 11.80$.

6-Amino-3- methyl-1,4- diphenyl-1,4- dihydropyrano [2,3-c] pyrazole-5carbonitrile (6a): Yield: $88 \%$; m.p.: $157-159^{\circ} \mathrm{C}$; IR $\left(\mathrm{KBr}, \mathrm{cm}^{-1}\right)$ v: 3480,3136 $\left(\mathrm{NH}_{2}\right), 3056\left(\mathrm{CH}\right.$ aromatic), $2020(\mathrm{CN}), 1642(\mathrm{C}=\mathrm{C}) ;{ }^{1} \mathrm{H}$ NMR $\left(\mathrm{DMSO}-d_{6}\right) \delta$ : $3.76\left(\mathrm{~s}, 3 \mathrm{H}, \mathrm{CH}_{3}\right), 6.69$ (s, $1 \mathrm{H}$, pyran $\left.\mathrm{H}-4\right), 5.34\left(\mathrm{~s}, 2 \mathrm{H}, \mathrm{NH}_{2}, \mathrm{D}_{2} \mathrm{O}\right.$ exchangeable), 7.26-7.36 (m, $\left.10 \mathrm{H}, 2 \mathrm{C}_{6} \mathrm{H}_{5}\right)$ ppm; Anal. calculated. for $\mathrm{C}_{20} \mathrm{H}_{16} \mathrm{~N}_{4} \mathrm{O}: \mathrm{C}, 73.17 ; \mathrm{H}$, 4.88; N, 17.07. Found: C, 73.22; H, 4.83; N, 16.89.

6-Amino-4- (4-methoxyphenyl)-3- methyl-1-phenyl-1,4-dihydro- pyrano [2,3c] pyrazole-5-carbonitrile (6b): Yield: $80 \%$; m.p.: $233-235^{\circ} \mathrm{C}$; IR $\left(\mathrm{KBr}, \mathrm{cm}^{-1}\right)$ v: 
3493-3358 $\left(\mathrm{NH}_{2}\right), 3058$ (CH aromatic), $2980\left(\mathrm{CH}\right.$ aliphatic), $2220(\mathrm{CN}) ;{ }^{1} \mathrm{H}$ NMR (DMSO- $\left.d_{6}\right) \delta: 2.88\left(\mathrm{~s}, 3 \mathrm{H}, \mathrm{CH}_{3}\right), 3.16\left(\mathrm{~s}, 3 \mathrm{H}, \mathrm{OCH}_{3}\right), 6.73(\mathrm{~s}, 1 \mathrm{H}$, pyran H4), 6.68 (s, 2H, $\mathrm{NH}_{2}, \mathrm{D}_{2} \mathrm{O}$ exchangeable), 7.26-7.39 (m, 9H, $\left.\mathrm{C}_{6} \mathrm{H}_{5}, \mathrm{C}_{6} \mathrm{H}_{4}\right) \mathrm{ppm}$; Anal. calculated. for $\mathrm{C}_{21} \mathrm{H}_{18} \mathrm{~N}_{4} \mathrm{O}_{2}$ : C, 70.39; H, 5.03; N, 15.64. Found: C, 70.42, $\mathrm{H}, 4.80, \mathrm{~N} 15.73$.

6-Amino-4-(4-chlorophenyl)-3-methyl-1-phenyl-1,4-dihydropyrano-[2,3c]pyrazole-5-carbonitrile (6c): Yield: 79\%; m.p.: $130-136{ }^{\circ} \mathrm{C} ; \mathrm{IR}\left(\mathrm{KBr}, \mathrm{cm}^{-1}\right)$ v: 3480-3328 $\left(\mathrm{NH}_{2}\right), 3058\left(\mathrm{CH}\right.$ aromatic), $2220(\mathrm{CN}), 1659(\mathrm{C}=\mathrm{N}), 1630(\mathrm{C}=\mathrm{C}) ;{ }^{1} \mathrm{H}$ NMR (DMSO- $\left.d_{6}\right) \delta: 4.74\left(\mathrm{~s}, 3 \mathrm{H}, \mathrm{CH}_{3}\right), 6.62(\mathrm{~s}, 1 \mathrm{H}$, pyran $\mathrm{H}-4), 6.85(\mathrm{~s}, 2 \mathrm{H}$, $\mathrm{NH}_{2}, \mathrm{D}_{2} \mathrm{O}$ exchangeable), 7.26-7.37 ( $\left.\mathrm{m}, 9 \mathrm{H},{ }^{-} \mathrm{C}_{6} \mathrm{H}_{5}, \mathrm{C}_{6} \mathrm{H}_{4}\right) \mathrm{ppm}$. Anal. calculated. for $\mathrm{C}_{20} \mathrm{H}_{15} \mathrm{ClN}_{4} \mathrm{O}: \mathrm{C}, 66.21 ; \mathrm{H}, 4.17 ; \mathrm{N}, 15.44$. Found: C, 66.30; H, $4.08 ; \mathrm{N}, 15.63$.

General procedure for the synthesis of the pyrano[2,3-c:6,5-c']dipyrazole derivatives $7 a-c$

To a solution of either compound $6 \mathrm{a}(3.28 \mathrm{~g}, 0.01 \mathrm{~mol}), 6 \mathrm{~b}(3.58 \mathrm{~g}, 0.01 \mathrm{~mol})$ or $6 \mathrm{c}(3.62 \mathrm{~g}, 0.01 \mathrm{~mol})$ in 1,4-dioxane $(40 \mathrm{ml})$ solution containing sodium acetate $(4.0 \mathrm{~g})$, hydrazine hydrate $(0.50 \mathrm{~g}, 0.01 \mathrm{~mol})$ was added. The reaction mixture was heated under reflux for $4 \mathrm{hr}$ then poured onto ice/water containing few drops of hydrochloric acid and the formed solid product, in each case, was collected by filtration.

5-Methyl-4,7- diphenyl-4,7- dihydro-1H- pyrano [2,3-c:6,5-c'] dipyrazol-3amine (7a): Yield: $73 \%$; m.p.: 103-105 ${ }^{\circ} \mathrm{C}$; IR $\left(\mathrm{KBr}, \mathrm{cm}^{-1}\right)$ v: 3486- $3350\left(\mathrm{NH}_{2}\right.$, $\mathrm{NH}), 3058\left(\mathrm{CH}\right.$ aromatic), $1650(\mathrm{C}=\mathrm{N}), 1636(\mathrm{C}=\mathrm{C}) ;{ }^{1} \mathrm{H}$ NMR (DMSO- $\left.d_{6}\right) \delta$ : $2.83\left(\mathrm{~s}, 3 \mathrm{H}, \mathrm{CH}_{3}\right), 6.56(\mathrm{~s}, 1 \mathrm{H}$, pyran $\mathrm{H}-4), 6.73\left(\mathrm{~s}, 2 \mathrm{H}, \mathrm{NH}_{2}, \mathrm{D}_{2} \mathrm{O}\right.$ exchangeable), $7.25-7.39\left(\mathrm{~m}, \quad 10 \mathrm{H}, \quad 2 \mathrm{C}_{6} \mathrm{H}_{5}\right), \quad 10.24\left(\mathrm{~s}, \quad 1 \mathrm{H}, \quad \mathrm{NH}, \quad \mathrm{D}_{2} \mathrm{O}\right.$ exchangeable) ppm. Anal. calculated. for $\mathrm{C}_{20} \mathrm{H}_{17} \mathrm{~N}_{5} \mathrm{O}: \mathrm{C}, 69.97 ; \mathrm{H}, 4.96 ; \mathrm{N}$, 20.41. Found: C, 69.77; H, 4.73; N, 20.69.

4-(4-Methoxyphenyl)-5- methyl-7- phenyl-4,7- dihydro-1H- pyrano [2,3c:6,5-c']dipyrazol-3-amine (7b): Yield: $78 \%$; m.p.: 136-139 ${ }^{\circ} \mathrm{C}$; IR $\left(\mathrm{KBr}, \mathrm{cm}^{-1}\right)$ v: 3458-3248 ( $\left.\mathrm{NH}_{2}, \mathrm{NH}\right), 3053$ (CH aromatic), 2987 (CH aliphatic), 1634 $(\mathrm{C}=\mathrm{C}) ;{ }^{1} \mathrm{H}$ NMR (DMSO-d $\left.d_{6}\right) \delta: 2.68\left(\mathrm{~s}, 3 \mathrm{H}, \mathrm{CH}_{3}\right), 3.11\left(\mathrm{~s}, 3 \mathrm{H}, \mathrm{OCH}_{3}\right), 6.24(\mathrm{~s}$, $2 \mathrm{H}, \mathrm{NH}_{2}, \mathrm{D}_{2} \mathrm{O}$ exchangeable), $6.60(\mathrm{~s}, 1 \mathrm{H}$, pyran $\mathrm{H}-4), 7.27-7.39\left(\mathrm{~m}, 9 \mathrm{H}, \mathrm{C}_{6} \mathrm{H}_{5}\right.$, $\left.\mathrm{C}_{6} \mathrm{H}_{4}\right), 8.27\left(\mathrm{~s}, 1 \mathrm{H}, \mathrm{NH}, \mathrm{D}_{2} \mathrm{O}\right.$ exchangeable) ppm; Anal. calculated. for $\mathrm{C}_{21} \mathrm{H}_{19} \mathrm{~N}_{5} \mathrm{O}_{2}$ : C, 67.56; H, 5.09; N, 18.77. Found: C, 67.80, H, 5.29; N, 8.83.

4-(4-Chlorophenyl)-5- methyl-7- phenyl-4,7- dihydro-1H- pyrano [2,3-c:6,5$c^{\prime}$ ] dipyrazol-3-amine (7c): Yield: $79 \%$; m.p.: 166-169 ${ }^{\circ} \mathrm{C}$; IR $\left(\mathrm{KBr}, \mathrm{cm}^{-1}\right)$ v: 3450-3236 $\left(\mathrm{NH}_{2}, \mathrm{NH}\right), 3058(\mathrm{CH}$ aromatic), $2988(\mathrm{CH}$ aliphatic $), 1650(\mathrm{C}=\mathrm{N})$, $1630(\mathrm{C}=\mathrm{C}) ;{ }^{1} \mathrm{H}$ NMR (DMSO-d 6 ) $\delta: 2.80\left(\mathrm{~s}, 3 \mathrm{H}, \mathrm{CH}_{3}\right), 6.64(\mathrm{~s}, 1 \mathrm{H}$, pyran H-4), 6.30 (s, 2H, $\mathrm{NH}_{2}, \mathrm{D}_{2} \mathrm{O}$ exchangeable), 7.24-7.38 (m, 9H, $\left.\mathrm{C}_{6} \mathrm{H}_{5}, \mathrm{C}_{6} \mathrm{H}_{4}\right), 8.32$ (s, $1 \mathrm{H}, \mathrm{D}_{2} \mathrm{O}$ exchangeable, $\mathrm{NH}$ ); Anal. calculated. for $\mathrm{C}_{20} \mathrm{H}_{16} \mathrm{ClN}_{5} \mathrm{O}$ : C, 63.58; $\mathrm{H}$, 4.24; N, 18.54. Found: C, 63.73; H, 4.35; N, 18.69.

Egypt. J. Chem. 58, No. 6 (2015) 
Amino-3-methyl-1- phenyl-4- (pyridin-3-yl)-1,4- dihydropyrano [2,3-c] pyrazole-5- carbonitrile (9)

To a solution of compound $1(1.74 \mathrm{~g}, 0.01 \mathrm{~mol})$ in ethanol $(40 \mathrm{ml})$ containing triethylamine $(0.50 \mathrm{ml})$, pyridine-3-aldehyde $(1.7 \mathrm{~g}, 0.01 \mathrm{~mol})$ and malononitrile $(0.66 \mathrm{~g}, 0.01 \mathrm{~mol})$ were added. The reaction mixture was heated under reflux for $2 \mathrm{hr}$ then left to cool and the formed solid product was collected by filtration and crystallized from ethanol.

Yield: 68\%; m.p.: $188-191^{\circ} \mathrm{C}$; IR $\left(\mathrm{KBr}, \mathrm{cm}^{-1}\right)$ v: 3396-3334 $\left(\mathrm{NH}_{2}\right), 3060(\mathrm{CH}$ aromatic), 2980, 2926 (CH aliphatic), $2220(\mathrm{CN}) ;{ }^{1} \mathrm{H}$ NMR (DMSO- $\left.d_{6}\right) \delta: 1.83$ (s, $\left.3 \mathrm{H}, \mathrm{CH}_{3}\right), 4.63$ (s, $1 \mathrm{H}$, pyran $\left.\mathrm{H}-4\right), 6.82$ (s, $2 \mathrm{H}, \mathrm{NH}_{2}, \mathrm{D}_{2} \mathrm{O}$ exchangeable), 7.30-8.39 (m, 9H, $\mathrm{C}_{6} \mathrm{H}_{5}$, pyridine $\left.\mathrm{H}\right)$ ppm; Anal. calculated. for $\mathrm{C}_{19} \mathrm{H}_{15} \mathrm{~N}_{5} \mathrm{O}: \mathrm{C}$, $69.30 ; \mathrm{H}, 4.56 ; \mathrm{N}, 21.28$. Found: C, 69.32; H 4.47; N , 21.39.

General procedure for the synthesis of compounds 11a-c

To a solution of compound $1(1.74 \mathrm{~g}, 0.01 \mathrm{~mol})$ in ethanol $(30 \mathrm{ml})$ containing triethylamine $\left(\begin{array}{llllll}1.0 & \mathrm{ml}\end{array}\right)$ either benzaldehyde $\left(\begin{array}{llll}1.08 & \mathrm{~g}, & 0.01 & \mathrm{~mol}\end{array}\right)$, 4methoxybenzaldehyde ( $1.36 \mathrm{~g}, 0.01 \mathrm{~mol})$ or 4-chlorobenzaldehyde $(1.42 \mathrm{~g}, 0.01$ mol) and 2-aminoprop-1-ene-1,1,3-tricarbonitrile $(1.32 \mathrm{~g}, 0.01 \mathrm{~mol})$ were added. The whole reaction mixture, in each case was heated under reflux for $1 \mathrm{hr}$ then left to cool then poured onto ice/water mixture containing few drops of hydrochloric acid. The formed solid product, in each case, was collected by filtration and crystallized from ethanol.

Diamino-3 -methyl- 1,4- diphenyl- 1,4 - dihydropyrazolo - [4',3':5,6]pyrano[2,3-b]pyridine-6-carbonitrile (11a): Yield: 83\%; m.p.: 166-169 ${ }^{\circ} \mathrm{C}$; IR $\left(\mathrm{KBr}, \mathrm{cm}^{-1}\right)$ v: 3393-3239 $\left(2 \mathrm{NH}_{2}\right), 3055(\mathrm{CH}$ aromatic $), 2932,2928(\mathrm{CH}$ aliphatic), $2220(\mathrm{CN}), 1650(\mathrm{C}=\mathrm{N}) ;{ }^{1} \mathrm{H}$ NMR (DMSO- $\left.d_{6}\right) \delta: 2.66\left(\mathrm{~s}, 3 \mathrm{H}, \mathrm{CH}_{3}\right)$, $4.58(\mathrm{~s}, 1 \mathrm{H}$, pyran $\mathrm{H}-4), 4.16,4.44\left(2 \mathrm{~s}, 4 \mathrm{H}, 2 \mathrm{NH}_{2}, \mathrm{D}_{2} \mathrm{O}\right.$ exchangeable), 7.28-7.73 (m, $10 \mathrm{H}, 2 \mathrm{C}_{6} \mathrm{H}_{5}$ ), Anal.calculated. for $\mathrm{C}_{23} \mathrm{H}_{18} \mathrm{~N}_{6} \mathrm{O}: \mathrm{C}, 70.05 ; \mathrm{H}, 4.57 ; \mathrm{N}, 21.32$. Found: C, 70.22; H, 4.78; N, 21.29 .

5,7-Diamino-4- (4-methoxyphenyl)-3- methyl-1-phenyl-1,4-dihydropyrazolo [4',3':5,6] pyrano[2,3-b]pyridine-6-carbonitrile (11b): Yield: 85\%; m.p.: 132$135{ }^{\circ} \mathrm{C}$; IR $\left(\mathrm{KBr}, \mathrm{cm}^{-1}\right)$ v: 3368-3263 $\left(2 \mathrm{NH}_{2}\right), 3056(\mathrm{CH}$ aromatic), 2953, 2912 (CH aliphatic), $2221(\mathrm{CN}), 1655(\mathrm{C}=\mathrm{N}) ;{ }^{1} \mathrm{H}$ NMR (DMSO- $\left.d_{6}\right) \delta: 2.83(\mathrm{~s}, 3 \mathrm{H}$, $\left.\mathrm{CH}_{3}\right), 3.13$ (s, $\left.3 \mathrm{H}, \mathrm{OCH}_{3}\right), 4.79$ (s, $1 \mathrm{H}$, pyran $\left.\mathrm{H}-4\right), 6.77,6.90\left(2 \mathrm{~s}, 4 \mathrm{H}, 2 \mathrm{NH}_{2}\right.$, $\mathrm{D}_{2} \mathrm{O}$ exchangeable), 7.26-7.86 (m, 9H, $\left.\mathrm{C}_{6} \mathrm{H}_{5}, \mathrm{C}_{6} \mathrm{H}_{4}\right)$. Anal. calculated. for $\mathrm{C}_{24} \mathrm{H}_{20} \mathrm{~N}_{6} \mathrm{O}_{2}$ : C, 67.92; H, 4.72; N, 19.81 . Found: C, 68.11; H, 4.80; N, 20.03.

5,7-Diamino-4- (4-chlorophenyl)-3- methyl-1- phenyl-1,4- dihydropyrazolo [4',3':5,6] pyrano[2,3-b]pyridine-6-carbonitrile (11c): Yield: 77 \%; m.p.: 190$193{ }^{\circ} \mathrm{C}$; IR $\left(\mathrm{KBr}, \mathrm{cm}^{-1}\right)$ v: 3479-3290 $\left(2 \mathrm{NH}_{2}\right), 3053(\mathrm{CH}$ aromatic), 2990, 2912 (CH aliphatic), $2220(\mathrm{CN}), 1633(\mathrm{C}=\mathrm{C}) .{ }^{1} \mathrm{H}$ NMR (DMSO- $\left.d_{6}\right) \delta: 2.78(\mathrm{~s}, 3 \mathrm{H}$, $\left.\mathrm{CH}_{3}\right), 4.60(\mathrm{~s}, 1 \mathrm{H}$, pyran $\mathrm{H}-4), 4.88,7.15\left(2 \mathrm{~s}, 4 \mathrm{H}, 2 \mathrm{NH}_{2}, \mathrm{D}_{2} \mathrm{O}\right.$ exchangeable), 7.27-7.82 (m, 9H, $\left.\mathrm{C}_{6} \mathrm{H}_{5}, \mathrm{C}_{6} \mathrm{H}_{4}\right)$, Anal. calculated. for $\mathrm{C}_{23} \mathrm{H}_{17} \mathrm{ClN}_{6} \mathrm{O}: \mathrm{C}, 64.41 ; \mathrm{H}$, 4.00; N, 19.60. Found: C, 64.66; H, 3.92; N 19.53.

Egypt. J. Chem. 58, No. 6 (2015) 
1-(5-Cyano-4-(4-methoxyphenyl)-3-methyl-1- phenyl-1,4- dihydropyrano [2,3-c]pyrazol-6-yl)-3-phenylthiourea (13)

To a solution of compound $6 \mathrm{~b}(3.58 \mathrm{~g}, 0.01 \mathrm{~mol})$ in ethanol $(40 \mathrm{ml})$ containing triethylamine $(1.0 \mathrm{ml})$, phenylisothiocyanate $(1.30 \mathrm{~g}, 0.01 \mathrm{~mol})$ was added. The reaction mixture was heated under reflux for $2 \mathrm{hr}$. The formed solid product was collected by filtration and crystallized from ethanol. Yield: $79 \%$; m.p.: 166-169 ${ }^{\circ} \mathrm{C}$; IR ( $\left.\mathrm{KBr}, \mathrm{cm}^{-1}\right)$ v: 3374, 3328 (2 NH), 3058 (CH aromatic), 2966, 2929 (CH aliphatic), 2220 (CN), $1180(\mathrm{C}=\mathrm{S}) ;{ }^{1} \mathrm{H}$ NMR (DMSO-d $) \delta: 2.76$ $\left(\mathrm{s}, 3 \mathrm{H}, \mathrm{CH}_{3}\right), 3.12\left(\mathrm{~s}, 3 \mathrm{H}, \mathrm{OCH}_{3}\right), 4.58(\mathrm{~s}, 1 \mathrm{H}$, pyran $\mathrm{H}-4), 7.28-7.08(\mathrm{~m}, 14 \mathrm{H}$, $\left.2 \mathrm{C}_{6} \mathrm{H}_{5}, \mathrm{C}_{6} \mathrm{H}_{4}\right), 8.28,8.32\left(2 \mathrm{~s}, 2 \mathrm{H}, 2 \mathrm{NH}, \mathrm{D}_{2} \mathrm{O}\right.$ exchangeable), Anal. calculated for $\mathrm{C}_{28} \mathrm{H}_{23} \mathrm{~N}_{5} \mathrm{O}_{2} \mathrm{~S}: \mathrm{C}, 68.15 ; \mathrm{H}, 4.67 ; \mathrm{N}, 14.20$. Found: C, 68.22; H, 4.82; N, 14.30.

5-Amino-1-methyl-3H-chromeno[4',3':4,5]pyrano[2,3-c ]pyrazol-6(11 b H)-one (14)

To a solution of compound $1(0.98 \mathrm{~g}, 0.01 \mathrm{~mol})$, salicylaldehyde $(1.23 \mathrm{~g}$, $0.01 \mathrm{~mol})$ in dioxane $(30 \mathrm{ml})$ containing triethylamine $(1.0 \mathrm{ml})$ and malononitrile $(0.66 \mathrm{~g}, 0.01 \mathrm{~mol})$ was added. The whole reaction mixture, was heated under reflux for $2 \mathrm{hr}$, left to cool then poured onto ice/water mixture containing few drops of hydrochloric acid. The formed solid product was collected by filtration and crystallized from ethanol. Yield: 83\%; m.p.: 166$169^{\circ} \mathrm{C}$; IR $\left(\mathrm{KBr}, \mathrm{cm}^{-1}\right)$ v: 3376, $3332\left(\mathrm{NH}_{2}\right), 3054(\mathrm{CH}$ aromatic), 2989, 2950 (CH aliphatic); ${ }^{1} \mathrm{H}$ NMR (DMSO- $\left.d_{6}\right) \delta: 2.76\left(\mathrm{~s}, 3 \mathrm{H}, \mathrm{CH}_{3}\right), 4.48(\mathrm{~s}, 1 \mathrm{H}$, pyran $\mathrm{H})$, $4.26\left(\mathrm{~s}, 2 \mathrm{H}, \mathrm{NH}_{2}, \mathrm{D}_{2} \mathrm{O}\right.$ exchangeable), 7.27-7.42 (m, 9H, $\mathrm{C}_{6} \mathrm{H}_{5}, \mathrm{C}_{6} \mathrm{H}_{4}$ ). Anal calculated. for $\mathrm{C}_{20} \mathrm{H}_{15} \mathrm{~N}_{3} \mathrm{O}_{3}$ : C, 69.56; H, 4.35; N, 12.17. Found: C, 69.30; H 4.22; $\mathrm{N}, 12.38$.

\section{Conclusions}

The present work showed the synthesis of pyrazole derivatives derived from 3-methyl-1-phenyl-1H-pyrazo 1-5(4H)-one. The synthesized compounds were screened against six cancer cell lines and the results showed that compounds $4 \mathrm{a}, 4 \mathrm{c}, 6 \mathrm{c}, 7 \mathrm{c}, 11 \mathrm{~b}, 11 \mathrm{c}$ and 13 are the most potent compounds.

\section{References}

1. Do“ mling, A., Wang, W. and Wang, K., Chemistry and biology of multicomponent reactions. Chem. Rev. 112, 3083-3135(2012).

2. Do“ mling, A., Recent developments in isocyanide based multicomponent reactions in applied chemistry. Chem. Rev. 106, 17-89(2006).

3. Moghaddam, F.M. and Boinee, H.Z., An efficient and facile one-step synthesis of highly substituted thiophenes. Tetrahedron, 60, 6085-6089(2004).

4. Molvi, K.I., Mansuri, M. and Sudarsanam, V. et al., Synthesis, anti-inflammatory, analgesic and antioxidant activities of some tetrasubstituted thiophenes. J. Enzyme Inhib. Med. Chem. 23, 829-838(2008) .

Egypt. J. Chem. 58, No. 6 (2015) 
5. Parai, M.K., Panda, G., Chaturvedi, V., Manju, Y.K. and Sinha, S., Thiophene containing triarylmethanesasantitubercularagents. Bioorg. Med. Chem. Lett. 18, 289292(2008).

6. Wardakhan, W., Abdel-Salam, O. and Elmegeed, G., Screening for antidepressant, sedative and analgesic activities of novel fused thiophene derivatives. Acta Pharm. 58, 114 (2008).

7. Sharma, S., Athar, F., Maurya, M.R. and Azam, A., Copper(II) complexes with substituted thiosemicarbazones. of thiophene-2-carboxaldehyde: synthesis, characterization and antiamoebic activity against E. histolytica. Eur. J. Med Chem. 40,1414-1419(2005).

8. William, O. F., Principles of Medicinal Chemistry, $3^{\text {rd }}$ ed., Lippincott Williams \& Wilkins Publication, Philadelphia (1989).

9. Sagardoy, A.A., Gil, M.J. and Villar, R., et al., Benzo[b]thiophene-6-carboxamide 1,1- dioxides: inhibitors of human cancer cell growth at nanomolar concentrations. Bioorg. Med. Chem. 18, 5701-5707(2010).

10. Fadda, A.A., Abdel-Latif, E. and El-Mekawy, R.E., Synthesis and molluscicidal activity of some new thiophene, thiadiazole and pyrazole derivatives. Eur. J. Med. Chem. 44, 1250-1256(2009).

11. Liu, X.H., Cui, P., Song, B.A., Bhadury, P.S., Zhu, H.L. and Wang, S.F., Synthesis, structure and antibacterial activity of novel 1-(5-substituted-3-substituted4,5-dihydropyrazol-1-yl)ethanone oxime ester derivatives. Bioorg. Med. Chem. 16, 4075-4082 (2008).

12. Ouyang, G., Chen, Z., Cai, X.J., Song, B.A., Bhadury, P.S., Yang, S., Jin, L.H., Xue, W., Hu, D.Y. and Zeng, S., Synthesis and antiviral activity of novel pyrazole derivatives containing oxime esters group. Bioorg. Med. Chem. 16, 9699-9707 (2008).

13. Abdel-Hafez, E.M.N., Rahma, G.E.A.A., Aziz, M.A., Radwan, M.F. and Farag, H.H., Design, synthesis and biological investigation of certain pyrazole-3-carboxylic acid derivatives as novel carriers for nitric oxide. Bioorg. Med. Chem. 17, 3829-3837 (2009).

14. Park, H.J., Lee, K., Park, S.J., Ahn, B., Lee, J.C., Cho, H.Y. and Lee, K.I., Identification of antitumor activity of pyrazole oxime ethers. Bioorg. Med. Chem. Lett. 15, 3307-3312 (2005).

15. Ouyang, G., Cai, X.J., Chen, Z., Song, B.A., Bhadury, P.S., Yang, S., Jin, L.H., Xue, W., Hu, D.Y. and Zeng, S., Synthesis and antiviral activities of pyrazole derivatives containing an oxime moiety. J. Agric. Food Chem. 56, 10160-10167 (2008).

16. Dai, H., Li, Y.Q., Du, D., Qin, X., Zhang, X., Yu, H.B. and Fang, J.X., Synthesis and biological activities of novel pyrazole oxime derivatives containing a 2-chloro-5thiazolyl moiety. J. Agric.Food Chem. 56, 10805-10810 (2008). 
17. Riyadh, S.M., Farghaly, T.A., Abdallah, M.A., Abdalla, M.M. and El-Aziz, M.R.A., New pyrazoles incorporating pyrazolylpyrazole moiety: Synthesis, anti-HCV and antitumor activity. Eur. J. Med.Chem. 45, 1042-1050 (2010).

18. Anzaldi, M., Maccio, C., Mazzei, M., Bertolotto, M., Ottonello, L., Dallegri, F. and Balbi, A., Antiproliferative and proapoptotic activities of a new class of pyrazole derivatives in HL-60 cells. Chem. Biodivers. 6, 1674-1687 (2009).

19. El-Shafei, A., Fadda, A.A., Khalil, A.M., Ameen, T.A.E. and Badria, F.A., Synthesis, antitumor evaluation, molecular modeling and quantitative structureactivity relationship (QSAR) of some novel arylazopyrazolodiazine and triazine analogs. Bioorg. Med. Chem. 17, 5096-5105 (2009).

20. Huang, K.H., Veal, J.M., Fadden, P.R., Rice, J.W., Eaves, J., Strachan, J.P., Barabasz, A.F., Foley, B.E., Barta, T.E. and Ma, W., Discovery of novel 2aminobenzamide inhibitors of heat shock protein 90 as potent, selective and orally active antitumor agents. J. Med. Chem. 52, 4288-4305 (2009).

21. Huang, K.H., Veal, J.M., Fadden, P.R., Rice, J.W., Eaves, J., Strachan, J.P., Barabasz, A.F., Foley, B.E., Barta, T. E., Ma, W., Discovery of novel 2aminobenzamide inhibitors of heat shock protein 90 as potent, selective and orally active antitumor agents. J. Med. Chem. 52, 4288-4305 (2009).

(Received 6/12/2015

accepted $16 / 12 / 2015$ 


\section{تثيييا ويناء ودراسة السمية لبعض المركبات الجديدة للبيرووزلات

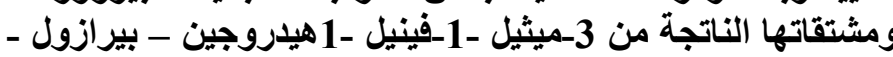 \\ 5

$$
\text { الهيئة القو مية للرّقابة و البحوث الدو ائية ص ب-29 القاهرة - مصر. }
$$

من خلال هذا البحث حاولنا بناء وتشييد بعض المركبات العضوية الحلقية الغير

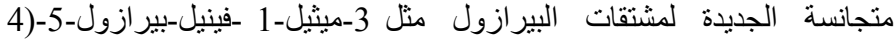
هيدروجين)-أون .

وقد تم تفاعل تللك المركبات مع الالدهيات الاروماتية وثيانو ميثيليين معا

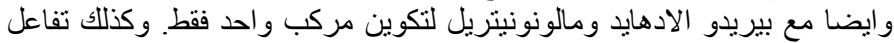

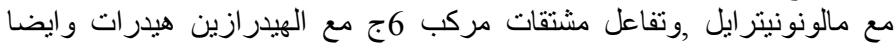

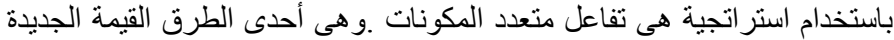

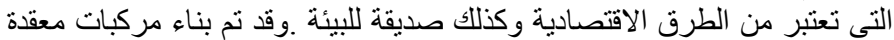

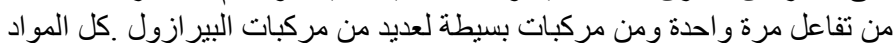

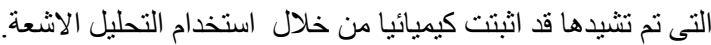

وقد خضعت تلك المركبات للتقبيم البيولوجى ودراسة مدى السمية لديها ضد إند الخلايا العادية وكذلك الخلايا الخبيثة. وقد نم قياس مدى السمية باستخدام طريقة

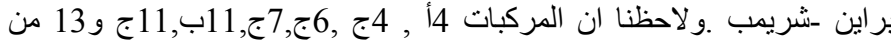

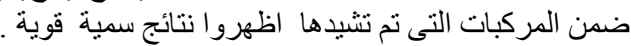

